\title{
Cancer screening in membranous nephropathy: How deep should we go? - A case report and literature review
}

\author{
Luís Mendonça ${ }^{1}$, Roberto Silva ${ }^{2}$, Ana Teresa Nunes ${ }^{1}$ \\ ${ }^{1}$ Serviço de Nefrologia, Centro Hospitalar Universitário São João, EPE, Porto, Portugal \\ 2 Serviço de Anatomia Patológica, Centro Hospitalar Universitário São João, EPE, Porto, Portugal
}

\section{ABSTRACT}

Membranous nephropathy is the most common cause of nephrotic syndrome in white adults. A non-negligible number of cases are associated with systemic conditions, namely cancer. We report the case of a 70-year-old man who presented with nephrotic syndrome and was diagnosed with membranous nephropathy. Although the initial screening did not detect any neoplastic lesion, additional exams revealed a nasopharyngeal carcinoma and a complete remission of the glomerular disease was observed after treating the cancer. Considering this very rare clinical association, we discuss the pitfalls underlying cancer screening in membranous nephropathy.

Keywords: Membranous nephropathy; cancer; nasopharyngeal carcinoma; screening.

\section{INTRODUCTION}

Membranous nephropathy ( $\mathrm{MN}$ ) is the most common cause of nephrotic syndrome in white adults, accounting for approximately $40 \%$ of all cases ${ }^{1}$. Although the discovery of an antibody targeting the podocyte phospholipase A2 type M receptor (anti-PLA2R) dramatically changed the diagnostic approach of this disease, a non-negligible number of cases (around 20\%) are associated with systemic conditions, namely cancer ${ }^{1,2}$. Here, we report a rare case of $\mathrm{MN}$ associated with a nasopharyngeal carcinoma and discuss the pitfalls underlying cancer screening in this glomerular disease.

\section{BRIEF CASE DESCRIPTION}

A 70-year-old man was sent by the assistant physician to our Nephrology outpatient clinic for a one-month history of lower limb edema and deterioration of renal function. The patient referred weight gain $(5 \mathrm{~kg})$, without any other complaint. He denied systemic symptoms such as fever, loss of appetite or fatigue.

His past medical history was remarkable for a long period of poorly controlled hypertension and he was an active heavy smoker (40 pack-years). He drank alcohol only on social occasions (around $30 \mathrm{~g}$ per month). He denied history of thromboembolic events. No history of renal disease was present in his family. His medication included furosemide $(80 \mathrm{mg})$, irbesartan $(300 \mathrm{mg})$, indapamide (2.5 $\mathrm{mg}$ ), amlodipine ( $5 \mathrm{mg}$ ) and simvastatin ( $20 \mathrm{mg}$ ). On physical exam, he was hypertensive $(170 / 100 \mathrm{mmHg})$ and with bilateral lower limb pitting edema below knee. His lab panel revealed decreased renal function (serum creatinine $1.5 \mathrm{mg} / \mathrm{dL}$ (1.2 mg/dL, 1 year earlier)) and slight hypoalbuminemia $(32.4 \mathrm{~g} / \mathrm{L}$ ). The urine sediment did not present leucocytes and erythrocytes. The twenty-four-hour urine presented nephrotic range proteinuria $(9.6 \mathrm{~g})$. The kidney ultrasound revealed normal sized kidneys with slight reduced corticomedullar differentiation.

A panel including antinuclear antibodies, anti-double stranded DNA, complement, anti-neutrophil cytoplasmic antibodies, immunofixation and Anti-PLA2R was negative. Hepatitis B, C and Human Immunodeficiency Virus infections were also excluded. A renal biopsy was performed. The optic microscopy, immunofluorescence and electronic microscopy were consistent with the diagnosis of membranous nephropathy. Chronic lesions of hypertensive nephroangiosclerosis were also evident. Electronic microscopy showed subepithelial deposits which were absent in the remaining glomerular compartments, namely subendothelium and mesangium. Inflammatory cells were not detected in glomeruli. IgG subclasses and tissue PLA2R immunofluorescence assays were not available at that time in our institution. No suspicious neoplastic lesion was detected on the upper and lower endoscopy study or the chest computed tomography (CT) scan. The prostate specific antigen (PSA) was normal. However, given the age, smoking habits of the patient and the negative anti-PLA2R, the CT scan was extended to cervical, abdominal and pelvic regions, showing a mass in nasopharynx. A surgical biopsy was performed, and the histologic examination was consistent with the diagnosis of squamous carcinoma (stage cT1N0). The patient was started on local radiotherapy. Three months after starting treatment, the carcinoma completely regressed and the patient went into complete remission of the nephrotic syndrome with sable glomerular filtration rate, normal serum albumin and proteinuria $<0.5 \mathrm{~g} /$ day.

\section{DISCUSSION}

There is no consistent definition for malignancy related $\mathrm{MN}$ as well as no formal algorithm for screening. The 2012 Kidney Disease Improving Global Outcomes (KDIGO) Guidelines for Glomerulonephritis recommend 
performing "appropriate investigations" to exclude secondary causes in all cases of biopsy-proven MN (not graded recommendation) ${ }^{3}$. On the same topic, the need for more studies investigating the most cost-effective cancer screening strategy is additionally recognized ${ }^{3}$.

The frequency of reported cancer in $\mathrm{MN}$ ranges from $5 \%$ to up more than $20 \%{ }^{4}$. In one of the largest series reported, Lefaucheur et al documented a global rate of $10 \%$ in a cohort of 240 patients with $\mathrm{MN}$, but reaching nearly $30 \%$ in men older than 65 years ${ }^{5}$. Carcinomas such as lung, breast, prostate and gastrointestinal system were the most frequent implicated lesions ${ }^{5}$. Age and type of tumor combined support the common practice of employing a screening workup close to that used in the general population, which includes an endoscopic study and mammography complemented by a chest X-ray / CT scan. This strategy can, however, miss the diagnosis as illustrated by our case. In the series reported by Lefaucheur et al, $21 \%$ of patients had a non-carcinoma neoplasia, including hematologic malignancies ${ }^{5}$. The clear correlation between cancer treatment, even in atypical locations, and $\mathrm{MN}$ remission underscores the importance of an appropriate stepwise screening when the suspicion is high ${ }^{5}$. Additionally, $\mathrm{MN}$ can be the first paraneoplastic manifestation of a cancer, allowing its early diagnosis and treatment, as observed in our case. In the series reported by Lefaucheur et al, metastasis were present in only $23 \%$ of the malignancies (excluding hematologic ones) ${ }^{5}$. The finding of a neoplasia also prevents the patient from being exposed to inappropriate and potential harmful immunosuppression.

Which factors increase the odds of MN associated with cancer? In which patients should a more thorough investigation be performed when the initial screening is negative? Some clinical, serologic and histopathologic findings that might help in this decision have been proposed. As referred above, age is an important indicator and the risk increases significantly in patients older than 65 years ${ }^{5}$. However, it should be emphasized that cancer incidence is increased in all age strata of MN patients, as compared to the general population ${ }^{6}$. The role of gender is less clear. While Birkeland and Storm found an excess of cancer in men with $\mathrm{MN}^{7}$, this association has not been confirmed in other series ${ }^{5}$. Regarding exposure, it has been clearly shown that the proportion of heavy smoking ( $\geq 20$ pack-years) is higher in patients with MN associated with neoplasia ${ }^{5}$, as documented in our patient. Pollution has been increasingly discussed as a hypothetical cause of $\mathrm{MN}^{8}$; however it is unexplored if it could be a common link between $\mathrm{MN}$ and cancer or if a high level of suspicion should be considered in patients with $\mathrm{MN}$ and a high pollution exposure level. Thromboembolic disease occurs in $25 \%$ of patients with cancer-associated $\mathrm{MN}$ and only $7 \%$ of patients with primary MN and so the occurrence of this complication also raises the suspicion of neoplasia ${ }^{9}$.

Some findings in renal tissue have also been described; however the association with malignancy related $\mathrm{MN}$ in not consistent across all studies. In the Lefaucheur et al series, an increased number of inflammatory cells infiltrating the glomeruli in patients with $\mathrm{MN}$ associated with cancer was shown, particularly if a threshold of eight or more cells was considered ${ }^{5}$. In contrast, Alnasrallah et al. find no association in their series; the median time from MN to cancer diagnosis was 40 months which suggests that some cases of MN were probably not cancer related ${ }^{6}$. The renal pattern of IgG subclasses has also been proposed as a biomarker. Some authors observed that IgG1 and IgG2 deposition was more intense in malignancy-related MN than primary $\mathrm{MN}^{10}$, although this was not replicated by others ${ }^{11}$. The lower deposition of IgG4 in these patients as compared to primary MN has also been reported and seems more consistent ${ }^{11,12}$. However, no reliable pattern of IgG subclasses is validated for clinical use and more research is needed ${ }^{13}$.

Since its discovery in 2009, anti-PLA2R has shown to be an excellent biomarker of MN, mainly as compared to other glomerular diseases. The high specificity and predictive positive value of this biomarker significantly increases the pre-test probability of primary MN (even potentially excluding the need of a renal biopsy for diagnosis is some cases) $)^{14}$. However, it does not definitely rule out the presence of cancer and several cases have been reported in patients with anti-PLA2R positive MN with a proportion up to $30 \%{ }^{15,16}$. However, some of these patients did not enter in remission after tumor excision, which does not exclude the presence of coincidental diseases ${ }^{17}$. So, in our opinion and in line with the literature, it is probably prudent to screen for cancer PLA2R positive patients until formal guidelines for the use of this biomarker are provided, although we are aware that the presence of this biomarker significantly increases the probability of primary MN. Since the discovery of a new autoantigen, Thrombospondin type-1 domain-containing 7A (THSD7A), some studies have found a higher incidence of cancer (approximately 20\%) in patients with $\mathrm{MN}$ associated with this biomarker, suggesting that THSD7A could be a useful indicator of malignancy-related $\mathrm{MN}^{18}$. However, again, this finding is not consistent in all series and more recently, other authors have reported relatively low rates of neoplasia ${ }^{17,19}$. The very recently described neural epidermal growth factor-like 1 protein (NELL-1) seems to be a biomarker of PLA2R negative primary $\mathrm{MN}$, but its value in other clinical grounds, including patients with cancer, still awaits more studies ${ }^{20}$.

The hypothetical mechanism by which neoplasia may be associated with $\mathrm{MN}$ relies mainly on the formation of antibodies against tumor antigens formed in situ or in circulation and trapped in glomerular membrane ${ }^{21}$. Accordingly, the definite pathophysiologic link between $\mathrm{MN}$ and cancer is the detection of tumor antigens in renal tissue as postulated by Cambier and Ronco ${ }^{22}$. However, most reported case series did not perform this investigation and future studies should consider this point.

As mentioned above, the evidence supporting a neoplasia screening workup in glomerular diseases is weak and there are no studies on cost-effectiveness, so it is difficult to implement a formal screening algorithm or score. Plaisier and Ronco have recently suggested a global strategy for cancer screening in glomerular diseases encompassing two steps: a routine screening (considering age and gender), and a targeted screening according to clinical and laboratorial data including symptoms, smoking history and anti-PLA2R. As observed in our case, the workup proposed by the authors implicitly underscores the importance of a high pre-test clinical suspicion derived from clinical and laboratorial clues ${ }^{9}$. That high level of clinical suspicion prompted us to perform an extended CT scan, although the most appropriate exam to search for an occult malignancy is not established in this context. A recent retrospective study has shown that $18 \mathrm{~F}-$ fluorodeoxyglucose positron emission tomography/CT could be an efficient way to detect an early neoplasia in patients with suspected 
paraneoplastic syndromes and further studies would be important in patients with $\mathrm{MN}^{23}$.

Every clinician should be alert for the possibility of cancer in all stages of management of a patient with membranous nephropathy, even in the long-term follow-up after clinical remission ${ }^{5,6,17}$. We think that, again, the level of initial clinical suspicion, the development of unexpected suspicious new symptoms and the lack of response to immunosuppression should all be considered to define the need for re-screening.

In conclusion, there is no reliable and definite clinicopathologic indicator of malignancy-related MN. Anti-PLA2R is currently an essential tool in the diagnosis of primary $\mathrm{MN}$, although it does not definitely rule out the presence of cancer. The proportion of cancer-associated $\mathrm{MN}$ increases with age, but the risk of neoplasia is increased in all patients with $\mathrm{MN}$ and for a long time after the diagnosis. The most cost-effective cancer screening workup in $\mathrm{MN}$ patients remains to be defined and a high level of clinical suspicion should be considered when the most frequent cancers are excluded, particularly in patients with anti-PLA2R negative. Malignancy treatment is essential for the control of the glomerular disease but, importantly, MN can also constitute an early paraneoplastic sign of a cancer still amenable for treatment as illustrated by our case.

\section{Disclosure of potential conflicts of interest: none declared}

\section{References}

1. Feehally J, Floege J, Tonelli M, Johnson RJ. Comprehensive clinical nephrology: Elsevier; 2019.

2. Beck LH, Jr., Bonegio RG, Lambeau G, Beck DM, Powell DW, Cummins TD, et al. M-type phospholipase $A 2$ receptor as target antigen in idiopathic membranous nephropathy. N Engl J Med. 2009;361(1):11-21.

3. Cattran DC, Feehally J, Cook HT, Liu ZH, Fervenza FC, Mezzano SA, et al. Kidney disease: improving global outcomes (KDIGO) glomerulonephritis work group. KDIGO clinical practice guideline for glomerulonephritis. Kidney Int Suppl. 2012;2(2):139-274.

4. Burstein DM, Korbet SM, Schwartz MM. Membranous glomerulonephritis and malignancy. Am J Kidney Dis. 1993;22(1):5-10.

5. Lefaucheur C, Stengel B, Nochy D, Martel P, Hill G, Jacquot C, et al. Membranous nephropathy and cancer: epidemiologic evidence and determinants of high-risk cancer association. Kidney Int. 2006;70(8):1510-7.
6. Alnasrallah B, Collins JF, Zwi LJ. Malignancy in Membranous Nephropathy: Evaluation of Incidence. Int J Nephrol. 2017;2017:8409829.

7. Birkeland SA, Storm HH. Glomerulonephritis and malignancy: a population-based analysis. Kidney Int. 2003;63(2):716-21.

8. Salant DJ. Unmet challenges in membranous nephropathy. Curr Opin Nephrol Hypertens. 2019;28(1):70-6.

9. Plaisier E, Ronco P. Screening for Cancer in Patients with Glomerular Diseases. Clin J Am Soc Nephrol. 2020;15(6):886-8.

10. Ohtani H, Wakui H, Komatsuda A, Okuyama S, Masai R, Maki N, et al. Distribution of glomerular IgG subclass deposits in malignancy-associated membranous nephropathy. Nephrol Dial Transplant. 2004;19(3):574-9.

11. Lonnbro-Widgren J, Ebefors K, Molne J, Nystrom J, Haraldsson B. Glomerular IgG subclasses in idiopathic and malignancy-associated membranous nephropathy. Clin Kidney J. 2015;8(4):433-9.

12. Qu Z, Liu G, Li J, Wu LH, Tan Y, Zheng X, et al. Absence of glomerular IgG4 deposition in patients with membranous nephropathy may indicate malignancy. Nephrol Dial Transplant. 2012;27(5):1931-7.

13. Murtas C, Ghiggeri GM. Membranous glomerulonephritis: histological and serological features to differentiate cancer-related and non-related forms. J Nephrol. 2016;29(4):469-78.

14. Floege J, Barbour SJ, Cattran DC, Hogan JJ, Nachman PH, Tang SCW, et al. Management and treatment of glomerular diseases (part 1): conclusions from a Kidney Disease: Improving Global Outcomes (KDIGO) Controversies Conference. Kidney Int. 2019;95(2):268-80.

15. Qin W, Beck LH, Jr., Zeng C, Chen Z, Li S, Zuo K, et al. Anti-phospholipase A2 receptor antibody in membranous nephropathy. J Am Soc Nephrol. 2011;22(6):1137-43.

16. Radice A, Pieruzzi F, Trezzi B, Ghiggeri G, Napodano P, D’Amico M, et al. Diagnostic specificity of autoantibodies to M-type phospholipase A2 receptor (PLA2R) in differentiating idiopathic membranous nephropathy (IMN) from secondary forms and other glomerular diseases. J Nephrol. 2018;31(2):271-8.

17. Zhang D, Zhang C, Bian F, Zhang W, Jiang G, Zou J. Clinicopathological features in membranous nephropathy with cancer: A retrospective single-center study and literature review. Int J Biol Markers. 2019:1724600819882698.

18. Hoxha E, Beck LH, Jr., Wiech T, Tomas NM, Probst C, Mindorf S, et al. An Indirect Immunofluorescence Method Facilitates Detection of Thrombospondin Type 1 Domain-Containing 7A-Specific Antibodies in Membranous Nephropathy. J Am Soc Nephrol. 2017;28(2):520-31.

19. Wang J, Cui Z, Lu J, Probst C, Zhang YM, Wang X, et al. Circulating Antibodies against Thrombospondin Type-I Domain-Containing 7A in Chinese Patients with Idiopathic Membranous Nephropathy. Clin J Am Soc Nephrol. 2017;12(10):1642-51.

20. Sethi S, Debiec H, Madden B, Charlesworth MC, Morelle J, Gross L, et al. Neural epidermal growth factor-like1 protein (NELL-1) associated membranous nephropathy. Kidney Int. 2019;97(1):163-74.

21. Monga D, Jhaveri KD. Glomerular Diseases and Cancer. American Society of Nephrology Onco-Nephrology Curriculum. 2016:1-9.

22. Cambier JF, Ronco P. Onco-nephrology: glomerular diseases with cancer. Clin J Am Soc Nephrol. 2012;7(10):1701-12

23. Sheikhbahaei S, Marcus CV, Fragomeni RS, Rowe SP, Javadi MS, Solnes LB. Whole-Body (18)F-FDG PET and (18)F-FDG PET/CT in Patients with Suspected Paraneoplastic Syndrome: A Systematic Review and Meta-Analysis of Diagnostic Accuracy. J Nucl Med. 2017;58(7):1031-6.

\section{Correspondence to:}

Luís Carlos Ferreira Mendonça

Centro Hospitalar Universitário São João

Alameda Professor Hernâni Monteiro, 4200 Porto, Portugal

E-mail: luiscfmendonca@gmail.com 